\title{
Neutron Diffraction Study of Self-Curing and Self-Crystallization Phenomena of Low-Temperature Dehydrogenating Products of Powder Crystals of Rare-Earth Metals Trihydroxides
}

\author{
Khidirov Irisali \\ Institute of Nuclear Physics of Uzbekistan, Tashkent, Uzbekistan. \\ Email: Khidirov@inp.uz \\ Received April $2^{\text {nd }}, 2013$; revised May $2^{\text {nd }}, 2013$; accepted May $9^{\text {th }}, 2013$ \\ Copyright (C) 2013 Khidirov Irisali. This is an open access article distributed under the Creative Commons Attribution License, which \\ permits unrestricted use, distribution, and reproduction in any medium, provided the original work is properly cited.
}

\begin{abstract}
The phenomenon of hydrogen thermoemission out of a crystal lattice of powder rare-earth metals trihydrooxides $\mathrm{R}(\mathrm{OH})_{3}(\mathrm{R}$ is La, $\mathrm{Pr}, \mathrm{Nd})$ was found. The hydrogen thermoemission out of a crystal lattice is partial or full removal of hydrogen out of the crystal lattice of powder hydrogen-containing crystal without change of symmetry of such crystal at continuous evacuation of high vacuum at evacuation temperature of $T_{\text {ev. }}$ which is lower than recrystallization $T_{\text {recrys. }}$ or disintegration $\left(\mathrm{T}_{\text {disinteg. }}\right)$ temperature of this crystal: $\mathrm{T}_{\text {ev. }}<\mathrm{T}_{\text {recrys. }}<\mathrm{T}_{\text {disineg. }}$. By neutron diffraction it is found that lowtemperature $\left(T_{\text {evacuation }}=400-420 \mathrm{~K}\right)$ removal of hydrogen (by hydrogen thermoemission) out of a crystal lattice of trihydrooxide $\mathrm{R}(\mathrm{OH})_{3}$ under continuous high vacuum evacuating makes possible to obtain metastable "trioxide" $\mathrm{R}[\mathrm{O}]_{3}$. Existence of such substance contradicts to the valence law (oxygen is bivalent and $\mathrm{Pr}$ is trivalent in hydroxides). Such "trioxide" has a superfluous negative charge: $\mathrm{R}^{3+} \mathrm{O}^{6-}$. So they aspire to "capture" three more protons (hydrogen ions) from a water molecules. Obviously, this substance can be stable at low temperatures and in the mediums, which are not containing hydrogen. In the air at room temperature this substance, most likely, interacting with water molecules, gradually again turns into trihydroxide $\mathrm{R}(\mathrm{OH})_{3}$, compensating the superfluous negative charge by three hydrogen atoms. From this it follows that substance $\mathrm{R}\left[\mathrm{O}_{3}\right]$ can simultaneously be an absorber of hydrogen and generator of oxygen at atmospheric conditions and in any mediums which contains water molecules, without any prior processing like heating or high pressure. Thus, the obtained material, without any prior processing like heating or high pressure, can simultaneously be oxygen generator and hydrogen accumulator in any mediums characteristic of $\mathrm{R}\left[\mathrm{O}_{3}\right]$ to transform into stable form $\mathrm{R}(\mathrm{OH})_{3}$ by selective bonding of hydrogen from the hydrogen-containing environment allowing implication of $\operatorname{Pr}\left[\mathrm{O}_{3}\right]$ as the hydrogen selective absorber. Separation (by low-temperature removal) of hydrogen out of $\mathrm{R}(\mathrm{OH})_{3}$ lattice can again lead to restoration of its capabilities to be a simultaneous hydrogen accumulator and oxygen generator in a medium containing water molecules.
\end{abstract}

Keywords: Hydrogen Termoemission; Rare-Earth Metals Trihydrooxides; Neutron Diffraction; High Vacuum;

Continuous Evacuation; Metastable "Trioxide" $\mathrm{R}\left[\mathrm{O}_{3}\right]$

\section{Introduction}

In the review work [1] a short report was given about the obtaining of metastable powder crystals of rare earth metal "trioxides" $\mathrm{R}\left[\mathrm{O}_{3}\right]$ (were $\mathrm{R}$ is $\mathrm{La}, \mathrm{Pr}, \mathrm{Nd}$ ) of the radical type by hydrogen thermoemission with the broken chemical bonds and not-coupled electrons. The hydrogen thermoemission out of a crystal lattice is partial or full removal of hydrogen out of the crystal lattice of powder hydrogen-containing crystal without change of symmetry of such crystal at continuous evacuation of high vacuum at evacuation temperature of $T_{\text {ev. }}$ which is lower than recrystallization $T_{\text {recrys. }}$ or disintegration $\left(T_{\text {disinteg. }}\right)$ temperatures of this crystal: $\mathrm{T}_{\text {ev. }}<\mathrm{T}_{\text {precrys. }}<\mathrm{T}_{\text {disineg. [1] }}$. Hydrogen atoms in a powder crystal lattice, having small nuclear weight, low binding energy and high diffusion rate, leave the lattice in continuously pumped-out high vacuum at relatively low temperaturewhich is lower than the temperatures of disintegration or recrystallisation. The configuration concerning relatively heavy atoms 
(earlier stabilized with hydrogen atoms) cannot change because of their insufficient diffusion mobility at this temperature. It is obvious that the metastable "trioxid" $\mathrm{R}\left[\mathrm{O}_{3}\right]$ obtained by hydrogen thermoemission has broken bonds, unpaired electrons and excessive negative charge $\mathrm{Rr}^{3+}\left[\mathrm{O}_{3}\right]^{6-}[1]$. It tends "trap" to three deficit protons (of a hydrogen ion) at the first possibility. This substance is stable only at relatively low temperature and in the medium free of hydrogen. In the air at room temperature, this substance, interacting with water molecules, gradually turns to trihyrdoxides $\mathrm{R}(\mathrm{OH})_{3}$ again, compensating excessive negative charge by means of three hydrogen atoms, trapping them from atmospheric water molecules, and releasing oxygen molecules. The aim of this work is to do more detailed study of the hydrogen thermoemission out of crystal lattice of powder rare earth metal (REM) trihydroxides $\mathrm{R}(\mathrm{OH})_{3}$, ( $\mathrm{R}$ is $\left.\mathrm{La}, \mathrm{Pr}, \mathrm{Nd}\right)$ and kinetics of self-curing and self-crystallisation of low-temperature dehydrogenating products of REM trihydroxides in atmospheric conditions at room temperature by neutron diffraction.

\section{Experiment Techniques}

The studied samples were obtained as a result of oxidation of the corresponding REM ( $\mathrm{La}, \mathrm{Pr}$ and $\mathrm{Nd}$ ) having 99.95\% purity in air at room temperature. The obtained samples were low-dispersed powder and the X-ray analysis showed that these samples contained only trihydroxides of $\mathrm{La}, \mathrm{Pr}$ and $\mathrm{Nd}$ with $\mathrm{UCl}_{3}$ - type hexagonal structure described in the frame of $\mathrm{P}_{3} / \mathrm{m}$ space group. Samples composition was determined by chemical analysis and by minimizing of the R-factors of structure determination based on neutron diffraction patterns. These results are consistent with the results of other authors [2,3]. All of them have lattice parameters close to each other, which are identical with values resulted in $[2,3]$.

Neutron diffraction experiment was carried out using the neutron diffractometer mounted at a thermal column of atomic reactor WWR-SM of the Institute of Nuclear Physics of Uzbekistan AS $(\lambda=0.1085 \mathrm{~nm})$. Calculation of structural characteristics was carried out by Rietveld full-profile method [4] using neutron diffraction data. X-ray diffraction patterns were obtained using the X-ray diffractometer DRON -3M $(\lambda=0.15418 \mathrm{~nm})$. Dehydrogenation of samples was carried in the SShVL-type vacuum furnace in continuously pumped high vacuum of not more than $5.33 \times 10^{-3} \mathrm{~Pa}$.

\section{Results and Discussion}

The neutron diffraction pattern of the initial sample $\mathrm{Pr}$ $(\mathrm{OH})_{3}$ is represented in Figure 1(a).

Diffraction patterns of other REM trihydroxides were similar to that of $\operatorname{Pr}(\mathrm{OH})_{3}$. Since according to [2] and data of the present work, all REMs $\mathrm{R}(\mathrm{OH})_{3}$ trihydrooxides having $\mathrm{UCl}_{3}$-type hexagonal structure (space group $\mathrm{P}_{3} / \mathrm{m}$ ) with lattice parameters close to each other, further in the text for an illustration we will use neutron diffraction pattern of one of them. The least R-factors on Bragg maxima at calculation of the neutron diffraction pattern by method of Ritveld were obtaining for the model in the frame of $\mathrm{P}_{3} / \mathrm{m}$ space group. The difference of experimental and calculated intensities of the neutron diffraction pattern of the $\operatorname{Pr}(\mathrm{OH})_{3}$ in the frame of $\mathrm{P}_{3} / \mathrm{m}$ space group is also presented in Figure 1(a). The structural characteristics of the $\mathrm{R}(\mathrm{OH})_{3}$ samples are given in Table 1. These results are quite in agreement with data of the work $[2,3]$. The strong incoherent background which is falling down with increase of Bragg angle (Figure 1(a) and see relatively the horizontal dotted line) is typical for the neutron diffraction patterns of $\mathrm{R}(\mathrm{OH})_{3}$. According to [5], only hydrogen nucleus causes the strong incoherent background strongly decreasing with growing Bragg angle because of large amplitude of incoherent scattering
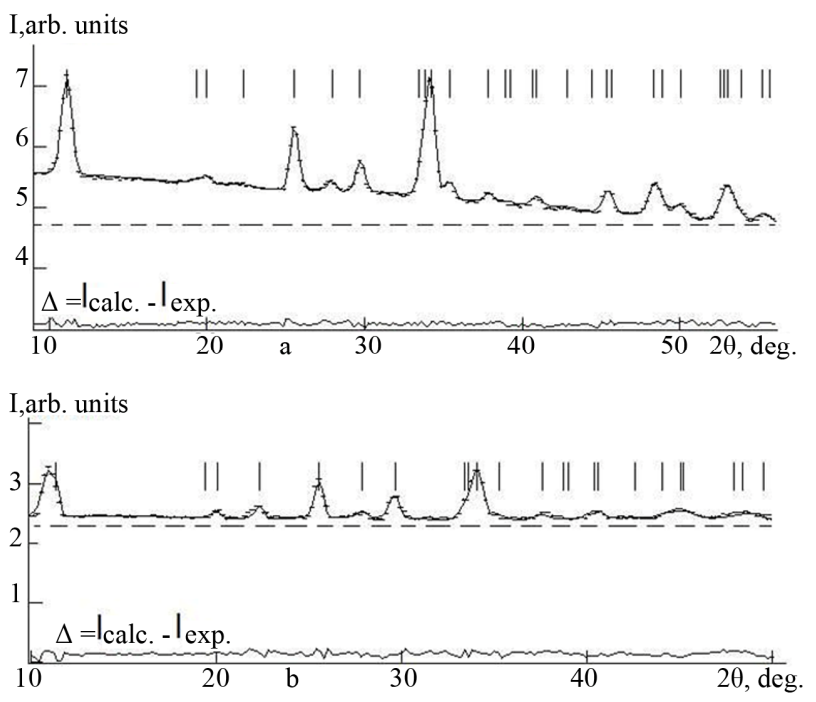

Figure 1. Neutron diffraction patterns of the praseodymium trihydroxid $\operatorname{Pr}(\mathrm{OH})_{3}$ (a) and the "trioxide" $\operatorname{Pr}\left[\mathrm{O}_{3}\right]$; (b) The dots are the neutron diffraction pattern data. The solid line is the calculated profile. $\Delta$ is the difference curve (experimental minus calculated). Above diffraction maxima were indicated calculated positions reflections $h k l$ in the frame space group $\mathrm{P6}_{3} / \mathrm{m}$.

Table 1. The structural characteristics of $\mathrm{R}(\mathrm{OH})_{3}$ samples.

\begin{tabular}{ccccc}
\hline Atom & Position & $\mathrm{x}$ & $\mathrm{y}$ & $\mathrm{z}$ \\
\hline $2 \mathrm{R}$ & $2(\mathrm{~d})$ & 0.6666 & 0.3333 & $1 / 4$ \\
$6 \mathrm{O}$ & $6(\mathrm{k})$ & $0.300 \pm 0.002$ & $0.385 \pm 0.002$ & $1 / 4$ \\
$6 \mathrm{H}$ & $6(\mathrm{k})$ & $0.160 \pm 0.003$ & $0.288 \pm 0.004$ & $1 / 4$ \\
\hline
\end{tabular}


of neutrons. Evacuation of hydrogen from lattice $\mathrm{R}(\mathrm{OH})_{3}$ began from the room temperature with the step of $25 \mathrm{~K}$ in continuously pumped out high vacuum in the vacuum furnace. Exposure time for each temperature at first was $24 \mathrm{~h}$. At dehydrogenating of the REM trihydroxides in the working volume of the furnace was provided at continuously pumped out to achieve the vacuum of not more than $5.33 \times 10^{-3} \mathrm{~Pa}$. After dehydrogenating at every temperature a neutron diffraction pattern was recorded and hydrogen quantity in samples was examined by decline of incoherent background caused by incoherent neutron scattering on hydrogen nuclei. The hydrogen content was also estimating by the analysis of the experimental and calculated neutron patterns intensities by Ritveld method (on minimization of the R-factors). Finally, after continuous vacuum evacuation of $\mathrm{R}(\mathrm{OH})_{3}$ at temperatures of $400-420 \mathrm{~K}$ during not less than $16 \mathrm{~h}$ (Figure 1(b)) in the neutron diffraction pattern of the samples did not contain a incoherent background which was falling down with increase of Bragg angle (see relatively to the horizontal dashed line at Figure 1(b)). It was first sign of absence of hydrogen in crystals. But previous diffraction patterns corresponding to the $\mathrm{P}_{3} / \mathrm{m}$ space group observed in neutron diffraction patterns of initial $\mathrm{R}(\mathrm{OH})_{3}$ remained, but their relation had changed.

Calculation of the neutron diffraction patterns of the samples after dehydrogenation at temperatures of 400 $420 \mathrm{~K}$ by the Ritveld method of the full profile analysis shows that the sample does not contain hydrogen, but crystal structures remains (Figure 1(b)). Essential reduction of the neutron diffraction intensity after dehydrogenation is the reason for, first of all, absence of hydrogen atoms in a lattice; secondly, smaller total quantity of dehydrogenation samples. The latter is connected to the fact that for acceleration of the process of hydrogen removal from the $\mathrm{R}(\mathrm{OH})_{3}$ powders three times less quantity of samples is taken than for initial $\mathrm{R}(\mathrm{OH})_{3}$ powders. After hydrogen removal, one can observe widening of halfwidth and distortion of diffraction peaks form, which is caused by pronounced deformations in crystal lattice after hydrogen removal. The best agreement between experimental and calculated intensities of neutron diffraction reflections (Figure 1(b)) and minimal errors in structure determination $(\mathrm{R})$ can be obtained only assumed that these crystal substances are "trioxides" having the chemical composition without hydrogen: $\left.\mathrm{R} \mathrm{O}_{3}\right]$. It is worth of mentioning that the temperature of hydrogen evacuation out of lattice for all $\mathrm{R}(\mathrm{OH})_{3}$ trihydroxides within errors in temperature determination $(\Delta T= \pm 12 \mathrm{~K})$ is almost the same. This can be explained by the fact that REM metals $\mathrm{La}$, Pr and Nd have similar valence electron shells and does not differ in sizes and masses of atoms, whereas all $\mathrm{R}(\mathrm{OH})_{3}$ trihydrooxides have isomorphic structure. The results of calculations of the substance $\operatorname{Pr}[\mathrm{O}]_{3}$ neutron diffraction pattern in the frame $\mathrm{P}_{3} / \mathrm{m}$ space group are presented in Table 2.

On should notice large errors in determination of the lattice parameters and large value of the $\mathrm{R}_{\mathrm{Br} \text {. }}$. It is apparently caused by distortion of diffraction peaks' forms due to strong statistical lattice deformations appearing after hydrogen removal. Formation of such a compound in the R-O systems contradicts to the valence conservation principle. Oxygen is double-valence, and REM can be either three- or four-valent. But all of them in $\mathrm{R}(\mathrm{OH})_{3}$ are three-valent. Apparently, it is necessary to assume that "trioxides" $\mathrm{R}\left[\mathrm{O}_{3}\right]$ have broken bonds and unpaired electrons, likewise that of radicals, that is they have excessive negative charge: $\mathrm{R}^{3+}\left[\mathrm{O}_{3}\right]^{6-}$. Apparently, therefore $\mathrm{R}^{3+}\left[\mathrm{O}_{3}\right]^{6-}$ have dead color and a sharp specific smell. Hydrogen thermoemission in powder crystals of rare earth metal trihydroxides $\mathrm{R}(\mathrm{OH})_{3}$ can be explained as relief of potential wells in crystals (Figure 2). Heavy atoms and hydrogen atoms have different initial wells. Hence to overcome the potential barrier less energy is required for hydrogen compared to heavier atoms. These substances are stable only at relatively low-temperatures and in media free of hydrogen. Indeed, annealing of $\left.\mathrm{R}_{0} \mathrm{O}_{3}\right]$ or vacuum evacuation of hydrogen out of lattice of $\mathrm{R}(\mathrm{OH})_{3}$ at temperature $420 \mathrm{~K}$ longer than 24 hours leads to its amorphisation, which is pointed by lack of selective diffraction reflections in neutron patterns and formation of small diffuse reflection at Bragg angles of $2 \theta=28-38$ degrees (Figure 3). Similar phenomenon takes place

Table 2. Structural characteristics and R-factors of the $\operatorname{Pr}\left[\mathrm{O}_{3}\right]$ "trioxide" in the model of space group $\mathrm{P6}_{3} / \mathrm{m}$.

\begin{tabular}{cccccc}
\hline \multirow{2}{*}{ Atom } & $\begin{array}{c}\text { Number } \\
\text { of atoms }\end{array}$ & Position & \multicolumn{3}{c}{ Coordinates } \\
\cline { 3 - 5 } & & $2(\mathrm{~d})$ & $2 / 3$ & $\mathrm{y}$ & $\mathrm{z}$ \\
\hline $\mathrm{Pr}$ & 2 & $6(\mathrm{k})$ & $0.376 \pm 0.002$ & $0.461 \pm 0.002$ & $1 / 4$ \\
$\mathrm{O}$ & 6 & & & \\
& \multicolumn{4}{c}{$\begin{array}{c}\mathrm{a}=0.658 \pm 0.018 \mathrm{~nm} ; \mathrm{c}=0.381 \pm 0.006 \mathrm{~nm} ; \\
\mathrm{R}_{\mathrm{p}}=1.4 \% ; \mathrm{R}_{\mathrm{wp}}=1.8 \% ; \mathrm{R}_{\mathrm{Br}}=9.4 \%\end{array}$} \\
\hline
\end{tabular}

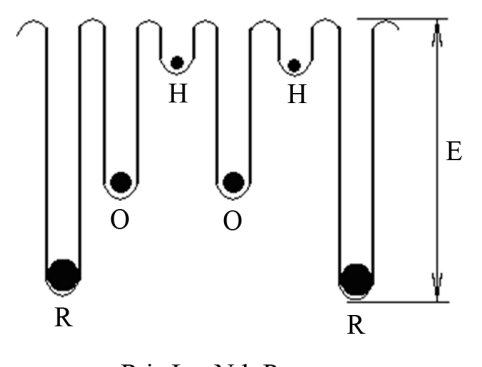

$\mathrm{R}$ is $\mathrm{La}, \mathrm{Nd}, \mathrm{Pr}$

Figure 2. The relief of potential wells in rare earth metal trihydroxides $\mathbf{R}(\mathrm{OH})_{3}$. 
I, arb. units

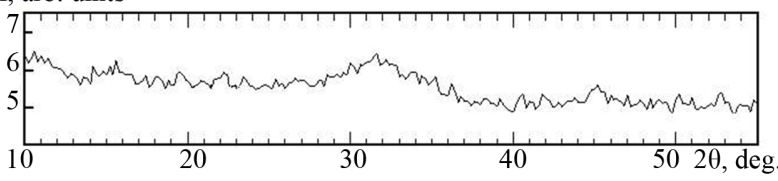

Figure 3. Neutron diffraction pattern of the amorphous $\operatorname{Pr}\left[\mathrm{O}_{3}\right]$.

at even slight increase of temperature up to $420 \mathrm{~K}$. The soaking of $\mathrm{R}(\mathrm{OH})_{3}$ trihydroxides or $\mathrm{R}\left[\mathrm{O}_{3}\right]$ "oxides" in continuously pumped out vacuum at temperature $\mathrm{T} \approx$ $470 \mathrm{~K}$ leads to escape both atoms of hydrogen and partially atoms of oxygen. At the same time recrystallization occurs and cubic oxide phases of corresponding REM is formed $\left(\mathrm{PrO}_{2-\mathrm{x}}\right.$ phase instead of $\operatorname{Pr}(\mathrm{OH})_{3}$ or $\mathrm{La}_{2} \mathrm{O}_{3}$ phase instead of $\mathrm{La}(\mathrm{OH})_{3}$ are formed (Figure 4). The diffraction patterns of the crystallines $\mathrm{R}\left[\mathrm{O}_{3}\right]$ samples after exposition in atmosphere at temperatures 285 - 290 $\mathrm{K}$ (the temperature in the reactor hall in winter) within 30 days are both and quantitatively become identical to the corresponding neutron diffraction patterns for the $\mathrm{R}(\mathrm{OH})_{3}$. Hence, one can conclude that in atmosphere the "trioxides" $\mathrm{R}\left[\mathrm{O}_{3}\right]$ "self-cures" until complete restoration of the REM trihydrooxides $\mathrm{R}(\mathrm{OH})_{3}$. Obviously, the reaction rate depends on temperature.

The self-curing of the "trioxides" $\mathrm{R}\left[\mathrm{O}_{3}\right]$ can be most likely explained as follows. The "trioxides" $\mathrm{R}\left[\mathrm{O}_{3}\right]$ due to its valence instability "tends" to trap three more protons. This can be accomplished by trapping in lattice of three hydrogen ions at first instance. Therefore, metastable rareeach metal "trioxides" in atmosphere, apparently, interact with water molecules, and retransform step by step back in the trihydrooxide $\mathrm{R}(\mathrm{OH})_{3}$, by compensating the excessive charge by three hydrogen atoms. This is demonstrated by the neutron diffraction analysis calculations of the product $\mathrm{R}\left[\mathrm{O}_{3}\right]$, taken in 30 days after obtaining of neutron diffraction patterns and by repeated formation of incoherent background on the neutron diffraction patterns. Since the hydrogen concentration on Earth's surface is rather low, one can assume that $\mathrm{R}[\mathrm{O}]_{3}$ captures hydrogen, mainly from, water vapors in the air, leaving oxygen molecules free:

$$
\begin{aligned}
& 4 \mathrm{R}^{3+}[\mathrm{O}]_{3}^{6-}(\text { powder metastable crystal })+6 \mathrm{H}_{2} \mathrm{O} \\
& =4 \mathrm{R}(\mathrm{OH})_{3}(\text { powder stable crystal })+3 \mathrm{O}_{2} \uparrow .
\end{aligned}
$$

If so, hence, "trioxide" $\mathrm{R}\left[\mathrm{O}_{3}\right]$ in atmospheric conditions can be simultaneously a hydrogen absorber and the oxygen generator. Separation (by low-temperature removal) of hydrogen out of $\mathrm{R}(\mathrm{OH})_{3}$ lattice can again lead to restoration of its capabilities to be a simultaneous hydrogen accumulator and oxygen generator in a medium containing water molecules. The cycle described above can be accomplished many times. It is shown in Figure 5.

It is useful to compare transformation rates of the "trioxides" $\mathrm{R}[\mathrm{O}]_{3}$ and the monoxides $\mathrm{ROOH}$ (by the way, obtained by dehydrogenation of the compositions $\mathrm{R}(\mathrm{OH})_{3}$ in air at the temperature of $\left.\mathrm{T} \sim 670 \mathrm{~K}[6]\right)$. The hydroxides $\mathrm{ROOH}$ even at short-term contact to air in atmospheric transform very quickly to corresponding $\mathrm{R}$ $(\mathrm{OH})_{3}$ trihydrooxides, that is caused by capture in air of water molecules by the monoxides $\mathrm{ROOH}$ without spliting into atoms [6]. It is possible to assume, that the "trioxides" $\mathrm{R}\left[\mathrm{O}_{3}\right]$ can "trap" hydrogen not only from water vapour, but also from other gaseous environment, having hydrogen partial pressure. Advantage of the "trioxides" $\mathrm{R}\left[\mathrm{O}_{3}\right]$ in comparison to other hydrogen-containing materials (for example, the titanium or intermetallic compounds) is that it absorbs hydrogen at small partial pressure and low temperature without an energy expense from outside. If the trihydroxide $\mathrm{R}(\mathrm{OH})_{3}$ to sustain at continuously pumped out vacuum at temerature of $\mathrm{T} \approx$ $420 \mathrm{~K}$ less, than $16 \mathrm{~h}$ then part of hydrogen in a lattice remains, which can be observed by the remained incoherent background in the neutron diffraction patterns. The analysis of the neutron pattern shows, that in these

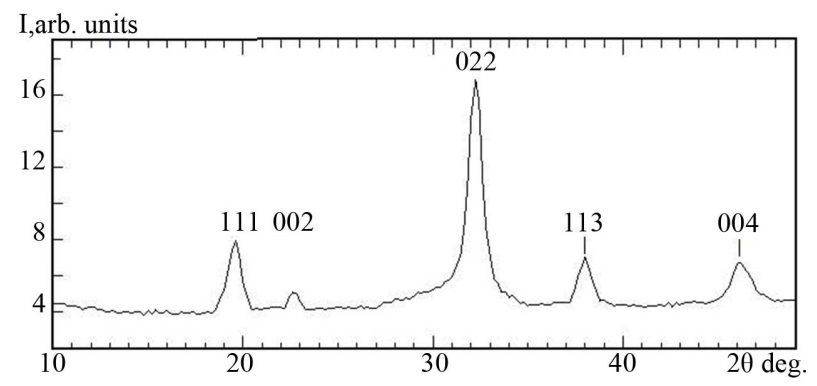

Figure 4. Neutron diffraction pattern of the $\mathrm{PrO}_{2-\mathrm{x}}$ (cubic phase, space group Fm3m). Above diffraction maxima were indicated Miller indexes $h k l$ in the frame of space group Fm3m.

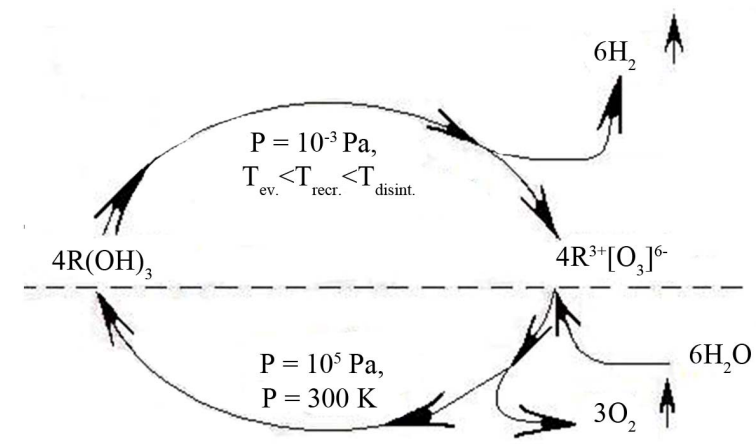

Figure 5. The scheme of the cycle of $\mathrm{R}\left[\mathrm{O}_{3}\right]$ production from $\mathrm{R}(\mathrm{OH})_{3}$ at continuously pumped high vacuum and repeated formation of $\mathrm{R}(\mathrm{OH})_{3}$ from "trioxide" $\mathrm{R}\left[\mathrm{O}_{3}\right]$ by self-curing in atmospheric conditions. 
conditions the strongly nonstochiometric hydroxides $\mathrm{R}\left[\mathrm{O}_{3} \mathrm{H}_{1}\right]$ are formed. For example, the structural characteristics and R-factors of determination of the structure for the substance $\operatorname{Pr}\left[\mathrm{O}_{3} \mathrm{H}_{1}\right]$ are given in Table 3. The soaking of these hydroxides nonstochiometric by hydrogen in atomspheric conditions for several days also leads to their "self-curing", that is to restoration of the complete trihydrooxides $\mathrm{R}(\mathrm{OH})_{3}$. The experiments revealed that amorphous $\mathrm{R}\left[\mathrm{O}_{3}\right]$ in atmosphere crystallize spontaneously and transform into trihydrooxides of corresponding rare earth metals during $1-1.5$ months at the temperature of the nuclear reactor hall in winter (285 $290 \mathrm{~K})$.

It was interesting to study the kinetics of this process. We therefore periodically generated the neutron diffracttion pattern of amorphous $\mathrm{La}\left[\mathrm{O}_{3}\right]$. During the measurements the sample was located continuously in the vanadium cylinder with diameter $6 \mathrm{~mm}$ and opened cap. Figure 6 shows some peculiar neutron diffraction patterns taken within 50 days. After evacuation of hydrogen out of lattice the $\mathrm{La}(\mathrm{OH})_{3}$ at temperature of $420 \mathrm{~K}$ for more than $24 \mathrm{~h}$ selective reflections disappear in neutron diffraction pattern and appear as both weak, and rather weak diffuse reflections. In the next 10 days weak diffraction maxima appear at Bragg angles $2 \theta=15^{\circ}$ and $19^{\circ}$, and intensity of diffuse reflections essentially grows (at Bragg angles $2 \theta=24^{\circ}-39^{\circ}$. After 15 days, weak selective reflections are formed on the diffuse maxima (Figure 6). The indicating shows that positions of these peaks correspond to the face-centered cubic oxide phase of $\mathrm{La}_{2} \mathrm{O}_{3}$ (space group $\mathrm{Fm} 3 \mathrm{~m}$ ). Hence, at the first stage in the amorphous phase the germs of the cubic oxide phase of $\mathrm{La}_{2} \mathrm{O}_{3}$ are formed. Further intensity of the reflections of the $\mathrm{La}_{2} \mathrm{O}_{3}$ oxide phase increases and diffuse reflection intensity decreases demonstrating that the formed oxide phase grows instead of the amorphous phase.

It is interesting to observe that on the 5th day after nucleation of the oxide phase, it disappear from the neutron diffraction pattern and one can observe some small diffraction maxima of the trihydroxide $\mathrm{La}(\mathrm{OH})_{3}$ (Figure 6). Indeed, according to [2,7], REM oxides $\mathrm{R}_{2} \mathrm{O}_{3}$, by ab-

Table 3. The structural characteristics and R-factors for the strongly nonstochiometric hydroxides $\mathrm{P}\left[\mathrm{O}_{3} \mathrm{H}_{1}\right]$ in the frame of $\mathrm{P6}_{3} / \mathrm{m}$ space group.

\begin{tabular}{cccccc}
\hline Atom & $\begin{array}{c}\text { Number } \\
\text { of atoms }\end{array}$ & Position & $\mathrm{X}$ & $\mathrm{y}$ & $\mathrm{z}$ \\
\hline $\mathrm{Pr}$ & 2 & $2(\mathrm{~d})$ & $2 / 3$ & $1 / 3$ & $1 / 4$ \\
$\mathrm{O}$ & 6 & $6(\mathrm{k})$ & $0.36 \pm 0.01$ & $0.44 \pm 0.01$ & $1 / 4$ \\
$\mathrm{H}$ & 1 & $6(\mathrm{k})$ & $0.16 \pm 0.09$ & $0.40 \pm 0.09$ & $1 / 4$ \\
\multicolumn{5}{c}{$\mathrm{a}=0.660 \pm 0.018 \mathrm{~nm} ; \mathrm{c}=0.382 \pm 0.02 \mathrm{~nm} ;$} \\
$\mathrm{R}$
\end{tabular}

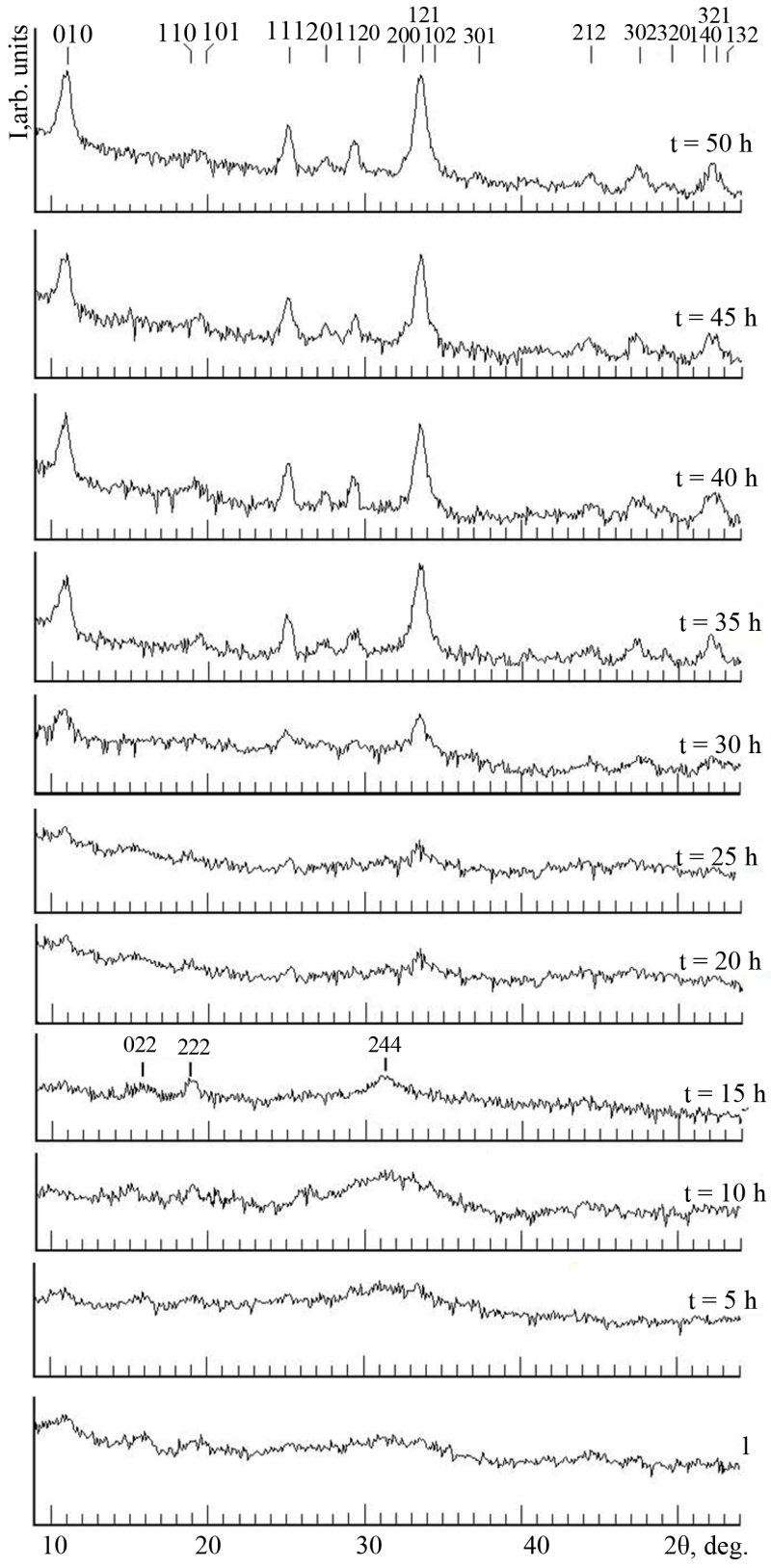

Figure 6. Neutron diffraction patterns of the amorphous $\mathrm{La}\left[\mathrm{O}_{3}\right]$ taken during spontaneous crystallization and its repeated transformation into $\mathrm{La}(\mathrm{OH})_{3}$ during various times: 1-amorphous $\mathrm{La}\left[\mathrm{O}_{3}\right]$; after its exposure in atmospheric conditions during times $t$.

sorbing a moisture from air, turn into to REM trihydroxides $\mathrm{R}(\mathrm{OH})_{3}$. In what follows the diffraction maxima of the trihydroxide phase $\mathrm{R}(\mathrm{OH})_{3}$ increase, and the diffuse reflexions decrease and finally disappear (Figure 6). In the beginning the diffraction reflections of the trihydrooxide $\mathrm{La}(\mathrm{OH})_{3}$ are strongly distorted, but finally they acquire ideal Gaussian form. Thus, duration of process of spontaneous crystallization and complete transformation of the amorphous $\mathrm{La}\left[\mathrm{O}_{3}\right]$ into the crystalline trihydrox- 
ide $\mathrm{La}(\mathrm{OH})_{3}$ in atmosphere at temperatures 285 - $290 \mathrm{~K}$ is approximately $\approx 30$ days. Certainly, the observed reaction rate depends on air temperature and humidity. To find out, how strong is such dependence on temperature we have sustained amorphous substance $\mathrm{La}\left[\mathrm{O}_{3}\right]$ in air at temperature $340 \mathrm{~K}$ within 2 hours. The neutron diffraction measurement after this endurance shows that the amorphous substance has already turned in badly generated $\mathrm{La}(\mathrm{OH})_{3}$ trihydroxide.

The author expresses sincere gratitude to Dr. Muslim Fazilov for the technical help.

\section{Conclusions}

Similar to being observed in powder interstitial solid solutions of the Ti-N-H, Zr-N-H and Ti-C-H systems [1,8-10], the hydrogen thermoemission phenomenon out of the crystal lattice of the chemical compounds of the rare-earth metal trihydroxides $\mathrm{R}(\mathrm{OH})_{3}$ is found. The hydrogen thermoemission out of a crystal lattice is partial or full removal of hydrogen out of a crystal lattice of a powder hydrogen-containing crystal without change of symmetry of a crystal at evacuation in continuously pumpedout high vacuum at evacuation temperature $T_{\text {ev. }}$. lower than recrystallization $T_{\text {recrys. }}$ or disintegration $\left(T_{\text {disinteg. }}\right)$ temperature of this crystal: $\mathrm{T}_{\mathrm{ev}}<\mathrm{T}_{\text {precrys. }}<\mathrm{T}_{\text {disineg. }}$. The phenomenon of hydrogen thermoemission in crystals can be observed only in powder samples due to simplification of hydrogen degasification, which has left a potential hole of a crystal lattice. As in the present stage of development materials technology, it is even possible to deal with nanocrystals and the hydrogen thermoemission can be used for obtaining new crystals in micro- and nanosizes.

In the work, according to the neutron diffraction structural analysis, metastable powder crystal rare earth "trioxides" $\mathrm{R}^{3+}\left[\mathrm{O}_{3}\right]^{6-}$ of radical type are received by hydrogen thermoemission at temperature of 400 - $420 \mathrm{~K}$ which are stable only at relatively low temperature which is lower than $\mathrm{T} \approx 400-420 \mathrm{~K}$ and in hydrogen-free environment. The substance $\mathrm{R}^{3+}\left[\mathrm{O}_{3}\right]^{6}$ possesses "self-curing" property grasping deficient hydrogen in environment containing hydrogen, and in atmospheric conditions, most likely, grasping hydrogen and releasing $\mathrm{O}_{2}$ from water molecules. Hence, the substance $\mathrm{R}\left[\mathrm{O}_{3}\right]$ simultaneously be hydrogen accumulator and oxygen generator in atmospheric conditions or in any environment containing water molecules without preliminary conditions (without heating and high pressure creation). The expenditure (by low-temperature removal) of hydrogen from $\mathrm{R}(\mathrm{OH})_{3}$ again leads to restoration of its property to simultaneously be the generator of oxygen and the hydrogen accumulator in the environment containing water molecules. The cycle described above can be accom- plished many times. The given cycle is outlined.

For powder crystals of rare earth metal trihydroxides three characteristic temperatures of hydrogen extraction out of their crystal lattice in continuously pumped-out high vacuum are determined:

1) The temperature of practically complete hydrogen evacuation out of a lattice without change in symmetry of a crystal $-\mathrm{T}_{\mathrm{ev}}(400-420 \mathrm{~K})$, at which a crystal structure does not change, but is significantly deformed;

2) The amorphisation temperature- $-T_{\text {amorp }}(450 \mathrm{~K})$ at which the selective diffraction reflections disappear and diffuse reflections appear in the neutron diffraction pattern;

3) The recrystallisation temperature $-\mathrm{T}_{\text {recr. }}(\approx 480 \mathrm{~K})$ at which cubic oxides phases corresponding to $\mathrm{RO}_{2-\mathrm{x}}$ or $\mathrm{La}_{2} \mathrm{O}_{3}$ form instead of the trihydroxides $\operatorname{Pr}(\mathrm{OH})_{3}$ and $\mathrm{La}(\mathrm{OH})_{3}$.

Strongly nonstochiometric rare earth metal hydroxides $\mathrm{R}\left[\mathrm{O}_{3} \mathrm{H}_{1}\right]$ are received from $\mathrm{R}(\mathrm{OH})_{3}$ by hydrogen thermoemission method and by that it is shown, that concentration of hydrogen in rare earth metal trihydroxides can be varied in a wide range up to full extraction of hydrogen out of a crystal lattice which possesses the property of self-curing in hydrogen-containing environment.

The interesting phenomenon consisting in spontaneous crystallisation of the amorphous substances $\mathrm{R}\left[\mathrm{O}_{3}\right]$ and its return transformation into the three hydroxides $\mathrm{R}(\mathrm{OH})_{3}$ is revealed in normal atmospheric conditions by selective absorption of a hydrogen from a water molecule or from a gaseous environment, having partial pressure of hydrogen. Duration of the observed process is about 35 days in atmospheric conditions at temperature of $285-290 \mathrm{~K}$.

\section{REFERENCES}

[1] I. Khidirov, "Neutron Diffraction Study of Hydrogen Thermoemission Phenomenon from Powder Crystals," In: I. Khidirov, Ed., Neutron Diffraction, Intechweb.org, Rijeka, 2012, pp. 155-178. http://dx.doi.org/10.5772/37597

[2] K. B. Portnoy and N. I. Timofeeva, "Oxygen Connection of Rare-Earth Metals," Metallurgiya, Moscow, 1986.

[3] I. Khidirov and V. T. Om, "Localization of hydrogen Atoms in Rare Earth Meral Trihydroxides $\mathrm{R}(\mathrm{OH})_{3}$,", Physica Status Solidi (a), Vol. 140, No. 2, 1993, pp. K59K62. http://dx.doi.org/10.1002/pssa.2211400231

[4] R. A. Young and D. B. Wiles, "Profile Scope Functions in Rietveld Refinements," Journal of Applied Crystallography, Vol. 15, No. 4, 1982, pp. 430-438. http://dx.doi.org/10.1107/S002188988201231X

[5] G. E. Bacon, "Neutron Diffraction," 3rd Edition, Clarendon Press, Oxford, 1975.

[6] P. V. Klevtcov and L. P. Sheina, "Hydrothermal Synthesis and Crystal Structure of Rare-Earth Elements Hydroxides," Russian Journal of Inorganic Materials, Vol. 1, 
No. 6, 1965, pp. 912-917.

[7] A. Arsenev, L. M. Kovba, X. S. Bogdasarov, B. F. Djurinskiy and A. V. Potemkin, "Compounds of the Rare Earth Elements. Systems with Oxides of the I-III Groups Elements," Nauka, Moscow, 1983.

[8] I. Khidirov, A. S. Rakhimov, V. E. Loryan and I. I. Kurbonov, "Obtaining of Metastable Phases in Ti-N and Zr-N Systems by Dehydrogenating of Solid Solutions," Russian Journal of Inorganic Materials, Vol. 26, No. 10, 1990, pp. 2113-2116.

[9] I. Khidirov, I. I. Kurbonov and A. Sh. Makhmudov, "De- termination of Crystals Stryctures of Metastable Ordered Phases in TiN and Zr-N Systems by Neutron Diffraction," Proceedings of the Moscov Interernational Composites Conference, Moscow, 14-16 November 1991, pp. 541545.

[10] I. Khidirov, B. B. Mirzaev, N. N. Mukhtarova, V. A. Bogolepov, A. F. Savenko and V. K. Pishuk, "Influence of Vacuum Thermal Treatment on Structure of Solid Solutions $\mathrm{TiC}_{\mathrm{x}} \mathrm{H}_{\mathrm{y}}$," Carbon Nanomaterials in Clean Energy Hydrogon Systems. Series C: Environmental Security, Springer, Dordrecht, 2008, pp. 679-684. 